Firas El-Baba, MD

Division of Pulmonary, Critical Care and Sleep

Medicine, Wayne State University School

of Medicine, Detroit, MI
Donovan Watza, PhD

Division of Pulmonary, Critical Care and Sleep

Medicine, Wayne State University School

of Medicine, Detroit, MI
Ayman 0. Soubani, MD

Professor of Medicine, Wayne State University

School of Medicine; Medical Director, Medical

ICU, Harper University Hospital; Service Chief,

Pulmonary and Critical Care, Karmanos Cancer

Center; Medical Director, Critical Care Service,

Karmanos Cancer Center, Detroit, MI

\title{
Q: Is Aspergillus isolated from respiratory cultures clinically significant?
}

\begin{abstract}
A: It depends on the patient's symptoms, underlying lung condition, immune status, and radiologic findings.

Because Aspergillus is ubiquitous, many patients have false-positive findings on respiratory culture and need no additional workup or treatment. But positive respiratory cultures may also indicate underlying serious lung disease. A thorough history to detect symptoms, underlying chronic lung disease, or an immunocompromising state followed by targeted laboratory tests and radiologic evaluation are adequate to ascertain the significance of this finding in the vast majority of patients.
\end{abstract}

See Editorial page 541

\section{THREE MAJOR GROUPS OF DISEASE}

Aspergillus is an environmentally ubiquitous and easily aerosolized mold encountered through daily exposure. ${ }^{1}$ Broadly, Aspergillusrelated lung diseases can be categorized into 3 major groups (Figure 1).

Allergic bronchopulmonary aspergillosis (ABPA) is an inflammatory lung condition caused by hypersensitivity reaction to Aspergillus antigens that almost exclusively occurs in patients with asthma or cystic fibrosis. ${ }^{2} \mathrm{Al}$ lergic reactions that do not fulfill the criteria for ABPA include Aspergillus sensitization and severe asthma with fungal sensitization.

Invasive pulmonary aspergillosis (IPA). IPA, unlike ABPA and chronic aspergillosis, is a severe, life-threatening, and often systemic disease process caused by Aspergillus species invading blood vessels, classically presenting in severely immunocompromised hosts and critically ill patients. ${ }^{3}$ A rare form of IPA is invasive Aspergillus tracheobronchitis.

doi:10.3949/ccjm.88a.20188
Chronic pulmonary aspergillosis is an umbrella term for a spectrum of disease patterns typically occurring in immunocompetent hosts with underlying lung diseases such as tuberculosis, chronic obstructive pulmonary disease, sarcoidosis, lung cancer, and lung radiation exposure and presenting with cavitary lesions that may progress slowly over time. ${ }^{4}$

\section{WHEN IS A POSITIVE CULTURE CLINICALLY SIGNIFICANT?}

Aspergillus infections, most commonly with A fumigatus and A flavus, account for approximately 15,000 hospitalizations and an estimated $\$ 1.2$ billion in hospital costs annually across the United States. ${ }^{5}$ Therefore, it is not uncommon for physicians to encounter an Aspergillus-positive respiratory culture in the clinical setting. This begets the question, Is the finding clinically significant?

In an adult patient without significant medical history, isolation of Aspergillus species in respiratory culture is likely a false-positive finding due to contamination or colonization of the respiratory flora by these ubiquitous fungal organisms. In hospitalized patients who undergo routine respiratory cultures, $80 \%$ to $90 \%$ of those with positive Aspergillus findings do not have significant aspergillosis lung disease. ${ }^{6,7}$ Even in patients with proven Aspergillus pulmonary infection, respiratory cultures are positive in $20 \%$ to $50 \%$ of patients, and as such the isolation of Aspergillus in respiratory cultures is neither sensitive nor specific in the diagnosis of most fungal respiratory infections and is not an integral part of the diagnostic criteria for Aspergillus-related lung diseases. ${ }^{8}$ Under these circumstances, in a patient who has no underlying lung disease and no immunocompromised state, we recommend obser-
Because

Aspergillus is ubiquitous, many patients will have false-positive findings on respiratory culture 


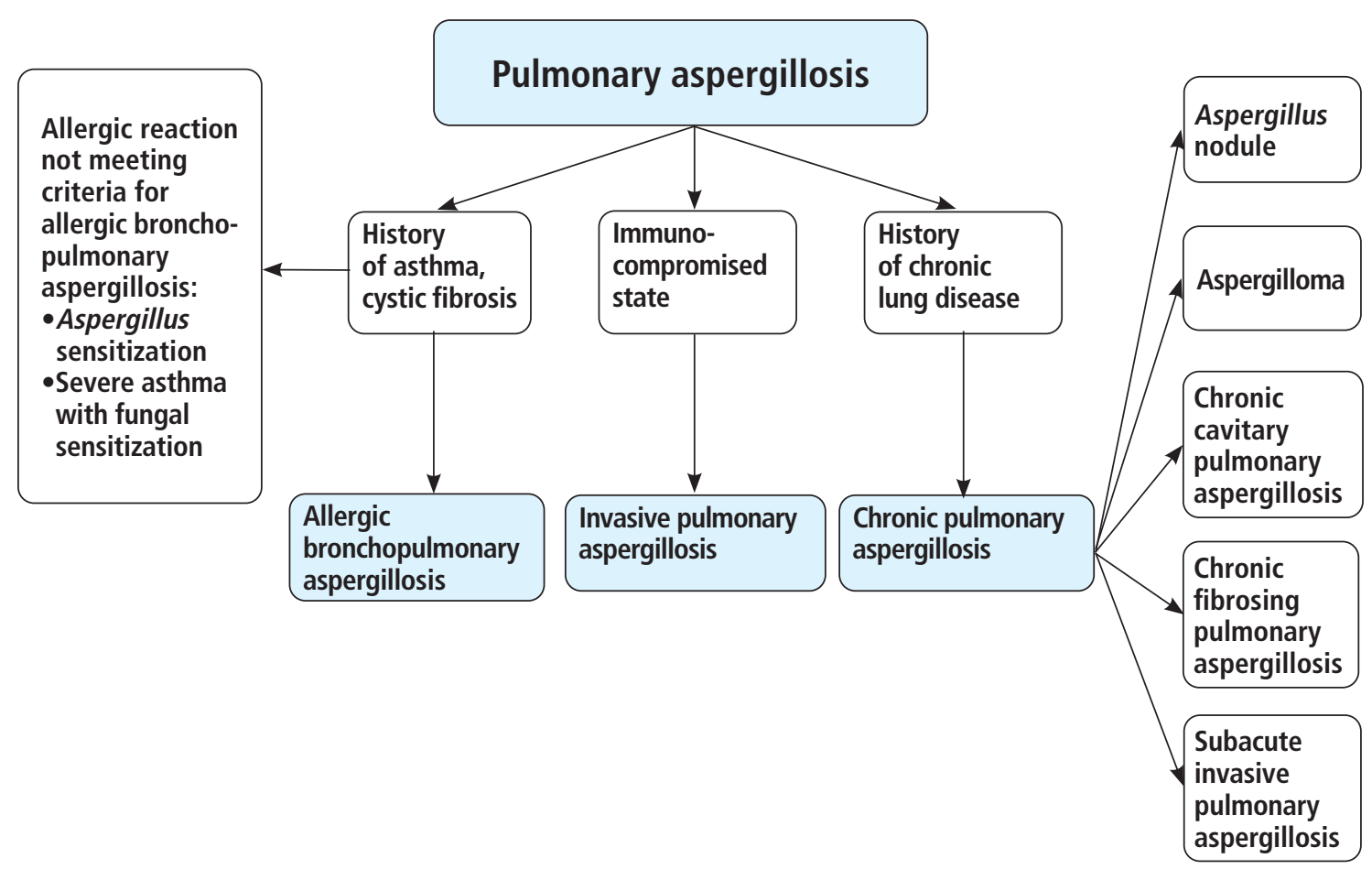

Figure 1. Pulmonary aspergillosis types, based on the patient's medical history.

vation and no further diagnostic or therapeutic intervention.

On the other hand, in a patient with respiratory symptoms, critical illness, underlying chronic lung disease, or an immunocompromising condition, detection of Aspergillus in respiratory culture may indicate underlying Aspergillus lung disease. ${ }^{3}$ In these situations, we recommend additional workup, and if the Aspergillus is proven to be the causative agent, then appropriate treatment should be started.

\section{THE HISTORY AND PHYSICAL}

It is imperative to assess the patient's history to quickly identify risk factors for pulmonary aspergillosis. We recommend first obtaining a thorough history and physical examination for all patients.

Key factors to consider include symptoms such as hemoptysis, chest pain, fever, and recent respiratory illness. Carefully assess for underlying chronic lung conditions including asthma, cystic fibrosis, chronic obstructive pulmonary disease, tuberculosis, lung surgery, radiation, pneumoconiosis, or sarcoidosis. In addition, a thorough evaluation should be done for conditions that may affect the immune system including leukemia, hematopoietic stem cell or solid-organ transplant, immunosuppressive therapy, and chronic corticosteroid therapy. ${ }^{3,5-10}$ In immunocompromised patients who present with sepsis and demonstrate tachypnea, tachycardia, fever, hypotension, and hypoxia, IPA should be considered, and rapid identification and treatment of the causative agent are crucial, as the mortality rate is high.

\section{LABORATORY TESTS AND IMAGING}

In patients with clinical presentations suggestive of aspergillosis, we suggest pairing a basic laboratory assessment (ie, a complete blood cell count) with radiographic imaging. Initial laboratory findings may narrow the differential diagnosis by identifying eosinophilia, which suggests ABPA, or severe neutropenia, which suggests IPA.

For imaging, we recommend high-resolution computed tomography (CT) of the chest rather than chest radiography to evaluate for Aspergillus-related lung disease, as it has superior ability to identify nodules, consolidation, cavitary lesions, and bronchiectasis. The finding of a cavitary lesion with or without intra- 
cavitary radiopacity suggests chronic aspergillosis, whereas the "halo" sign or "air crescent" sign suggests IPA (Figure 2), and bronchiectasis is seen in patients with ABPA. ${ }^{11}$ In evaluating chest CT findings, it is always useful to compare against previous imaging results and to consider other conditions that may coexist with positive Aspergillus in the respiratory sample.

\section{Galactomannan and beta-D-glucan}

In patients with risk factors and suspicious imaging findings, we recommend next testing for the serologic markers galactomannan and beta-D-glucan.

The specificity and sensitivity of these tests in the diagnosis of IPA depend on the host and cutoff value. When a cutoff assay index of 0.5 is used, the combined sensitivity for serum galactomannan has been calculated as $74 \%$ (95\% confidence interval [CI] 64-82) and its sensitivity as $85 \%$ (95\% CI 77-90). Serum beta-D-glucan had a sensitivity of $81 \%$ (95\% CI 73-87) and specificity of 61\% (95\% CI 46-75). ${ }^{10}$

The detection of galactomannan in bronchoalveolar lavage fluid is more sensitive and specific in the diagnosis of IPA, with a combined sensitivity of 79\% (95\% CI 65-88) and specificity of $84 \%$ (95\% CI 74-91). The procedure is relatively safe and should be considered in patients who have risk factors or have significant radiologic findings that suggest Aspergillus lung disease.

If the clinical or radiologic picture suggests ABPA, measuring serum total and Aspergillusspecific immunoglobulin E levels is needed to confirm the diagnosis.

\section{Biopsy is the gold standard but rarely needed}

The gold standard for diagnosis of most cases of Aspergillus-related lung disease is surgical biopsy and histopathologic confirmation. Unfortunately, biopsy often cannot be done owing to concomitant pulmonary comorbidities, severe immunocompromise, or critical illness with respiratory failure. Innovations in bronchoscopic procedures for microbiologic and pathologic samples, coupled with advances in radiology and Aspergillus biomarkers, have significantly reduced the need for surgical lung biopsy in these patients.

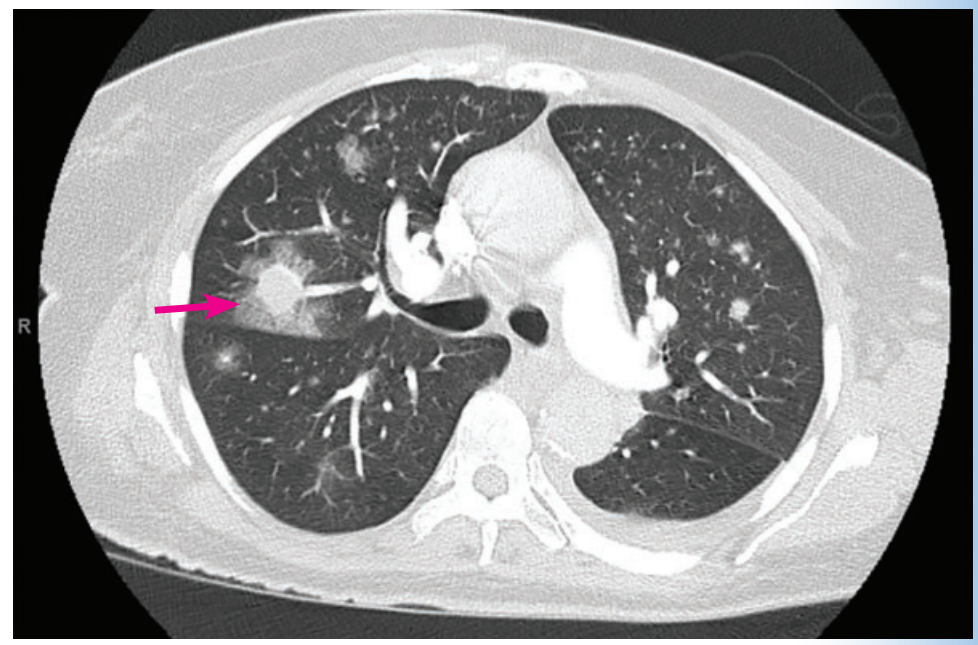

Figure 2. Computed tomography shows multiple pulmonary nodules, some surrounded by ground-glass changes consistent with the "halo" sign (arrow) in a patient with invasive pulmonary aspergillosis.

\section{MANAGEMENT}

Management depends on the Aspergillus-related diagnosis and the patient's clinical status. When considering conditions such as ABPA or chronic aspergillosis, we suggest waiting until the diagnosis is confirmed before initiating treatment.

However, IPA is more rapidly progressive and has a high mortality rate. Therefore, if clinical suspicion is high, therapy should not be delayed for the establishment of the diagnosis of proven or probable disease. In these situations, we suggest starting empiric therapy with a triazole agent while waiting for the results of cultures and biomarkers.

\section{ALWAYS CONSIDER THE CLINICAL PICTURE}

Due to the ubiquity of Aspergillus, many patients have false-positive findings on respiratory culture and require no additional workup or treatment. However, Aspergillus-positive respiratory cultures may be an indication of underlying serious Aspergillus lung disease. A thorough history to detect symptoms, underlying chronic lung disease, or immunocompromising state, followed by targeted laboratory tests and radiologic evaluation, is adequate to ascertain the significance of this finding in most patients.

\section{DISCLOSURES}

The authors report no relevant financial relationships which, in the context of their contributions, could be perceived as a potential conflict of interest. 


\section{REFERENCES}

1. Hospenthal DR, Kwon-Chung KJ, Bennett JE. Concentrations of airborne Aspergillus compared to the incidence of invasive aspergillosis: lack of correlation. Med Mycol 1998; 36(3):165-168. pmid:9776829

2. El-Baba F, Gao Y, Soubani AO. Pulmonary aspergillosis: what the generalist needs to know. Am J Med 2020; 133(6):668-674. doi:10.1016/j.amjmed.2020.02.025

3. Manuel RJ, Kibbler CC. The epidemiology and prevention of invasive aspergillosis. J Hosp Infect 1998; 39(2):95-109. doi:10.1016/s0195-6701(98)90323-1

4. Smith NL, Denning DW. Underlying conditions in chronic pulmonary aspergillosis including simple aspergilloma. Eur Respir J 2011; 37(4):865-872. doi:10.1183/09031936.00054810

5. Benedict K, Jackson BR, Chiller T, Beer KD. Estimation of direct healthcare costs of fungal diseases in the United States. Clin Infect Dis 2019; 68(11):1791-1797. doi:10.1093/cid/ciy776

6. Soubani AO, Khanchandani G, Ahmed HP. Clinical significance of lower respiratory tract Aspergillus culture in elderly hospitalized patients. Eur J Clin Microbiol Infect Dis 2004; 23(6):491-494. doi:10.1007/s10096-004-1137-1

7. Treger TR, Visscher DW, Bartlett MS, Smith JW. Diagnosis of pulmo- nary infection caused by Aspergillus: usefulness of respiratory cultures. J Infect Dis 1985; 152(3):572-576. doi:10.1093/infdis/152.3.572

8. Fayemiwo S, Moore CB, Foden P, Denning DW, Richardson MD. Comparative performance of Aspergillus galactomannan ELISA and PCR in sputum from patients with ABPA and CPA. J Microbiol Methods 2017; 140:32-39. doi:10.1016/j.mimet.2017.06.016

9. Haydour Q, Hage CA, Carmona EM, et al. Diagnosis of fungal infections. a systematic review and meta-analysis supporting American Thoracic Society Practice guideline. Ann Am Thorac Soc 2019; 16(9):1179-1188. doi:10.1513/AnnalsATS.201811-766OC

10. Hage CA, Carmona EM, Epelbaum O, et al. Microbiological laboratory testing in the diagnosis of fungal infections in pulmonary and critical care practice. An official American Thoracic Society Clinical Practice guideline. Am J Respir Crit Care Med 2019; 200(5):535-550. doi:10.1164/rccm.201906-1185ST

11. Prasad A, Agarwal K, Deepak D, Atwal SS. Pulmonary aspergillosis: what CT can offer before it is too late! J Clin Diagn Res 2016; 10(4):TE01-TE5. doi:10.7860/JCDR/2016/17141.7684

Address: Ayman O. Soubani, MD, Critical Care Service, Karmanos Cancer Center, 3990 John R Street, 3 Hudson, Detroit, MI 48201;

asoubani@med.wayne.edu 